\title{
ON COMPLETE INTEGRAL CLOSURE AND ARCHIMEDEAN VALUATION DOMAINS
}

\author{
ROBERT GILMER
}

(Received 19 October 1995)

Communicated by B. A. Davey

\begin{abstract}
Suppose $D$ is an integral domain with quotient field $K$ and that $L$ is an extension field of $K$. We show in Theorem 4 that if the complete integral closure of $D$ is an intersection of Archimedean valuation domains on $K$, then the complete integral closure of $D$ in $L$ is an intersection of Archimedean valuation domains on $L$; this answers a question raised by Gilmer and Heinzer in 1965 .
\end{abstract}

1991 Mathematics subject classification (Amer. Math. Soc.): primary 13A18, 13B02; secondary 13B22, $13 \mathrm{G} 05$.

All rings considered in this paper are assumed to be commutative and to contain a unity element. If $R$ is a subring of $S$, we assume that the unity element of $S$ belongs to $R$, and hence is the unity element of $R$.

If $R$ is a subring of $S$ and $s \in S$, then as is well known, $s$ is integral over $R$ if and only if $R[s]$ is a finitely generated $R$-module. On the other hand, if $R[s]$ is contained in a finitely generated $R$-submodule of $S$, then $s$ is said to be almost integral over $R$. Clearly $s$ is almost integral over $R$ if it is integral over $R$; the converse holds if $R$ is Noetherian, but not in general. We denote by $C(R, S)$ the set of elements of $S$ that are almost integral over $R ; C(R, S)$ is a subring of $S$ containing $R$, and is called the complete integral closure of $R$ in $S$. If $C(R, S)=S$, we say that $S$ is almost integral over $R$, and at the opposite extreme where $C(R, S)=R$, we say that $R$ is completely integrally closed in $S$. If $S$ is the total quotient ring of $R$, then $C(R, S)$ is the complete integral closure of $R$, and if $C(R, S)=R$, then $R$ is said to be completely integrally closed.

The concept of almost integrality was first considered by Krull in his famous 1932 paper Allgemeine Bewertungstheorie [5], in which he introduced the notion

(C) 1996 Australian Mathematical Society 0263-6115/96 \$A2.00+0.00 
of a general valuation and proved, among other things, that an integral domain is integrally closed if and only if it is an intersection of valuation domains [5, Satz 7]. (More precisely, Krull considers the concept in the case where $R$ is an integral domain and $S$ is the quotient field of $R$. In this case it is easy to show that $s \in S$ is almost integral over $R$ if and only if there exists a nonzero element $r \in R$ such that $r s^{k} \in R$ for each positive integer $k$.) Krull also proved [5, Satz 8] that a valuation domain $V$ is completely integrally closed if and only if the rank of $V$ is at most 1. (More generally, the complete integral closure of $V$ is either $V_{P}$ (if $V$ has a height-one prime ideal $P$ ) or the quotient field $F$ of $V$ (if $V$ has no height-one prime).) This led Krull to conjecture [5, p. 170] that a domain is completely integrally closed if and only if it is an intersection of valuation domains of rank at most 1 - that is, of Archimedean valuation domains. Nakayama disproved Krull's conjecture in $[7,8]$, and a simpler counterexample due to J. Ohm appears as Example 19.12 of [2]. On the other hand, Krull's conjecture is known to be correct for certain classes of domains; for example, it is valid for Noetherian domains [6, Theorem 33.10] and for domains of finite character [3, Proposition 5], [1, Theorem 5]. This paper answers a question related to the discussion above that arose in work that W. Heinzer and I did in connection with [3]. The question is the following:

Suppose $D$ is an integral domain with quotient field $K$, and assume that the complete integral closure of $D$ is an intersection of Archimedean

(Q) valuation domains on $K$. If $L$ is an extension field of $K$, is the complete integral closure of $D$ in $L$ an intersection of Archimedean valuation domains on $L$ ?

We show in Theorem 4 that (Q) has an affirmative answer. Our general reference for results concerning almost integrality is Section 13 of [2]. We first resolve the case of (Q) where $L$ is algebraic over $K$; Lemma 1 and Theorem 2 deal with the algebraic case.

LEMMA 1. Let $\left\{D_{a}\right\}_{a \in A}$ be a family of integrally closed domains with common quotient field $K$, and assume that $D=\bigcap_{a \in A} D_{a}$ also has quotient field $K$. Assume that $L$ is an algebraic extension field of $K$, and use bars to denote integral closure in L. Then $\bar{D}=\bigcap_{a \in A} \overline{D_{a}}$.

PROOF. The inclusion $\bar{D} \subseteq \bigcap_{a} \overline{D_{a}}$ is clear. Suppose $t \in \bigcap \overline{D_{a}}$ and let $f(x)$ be the minimal polynomial for $t$ over $K$. Since $D_{a}$ is integrally closed for $a \in A$, it is known that $f(x) \in D_{a}[x]\left[2\right.$, Theorem 10.5], and hence $f(x) \in \bigcap_{a \in A} D_{a}[x]=$ $\left(\bigcap_{a} D_{a}\right)[x]=D[x]$. Therefore $t \in \bar{D}$, as we wished to show. 
THEOREM 2. Let $D$ be an integral domain with quotient field $K$ and let $L$ be an algebraic extension field of $K$. If the complete integral closure $E$ of $D$ is an intersection of Archimedean valuation domains on $K$, then the complete integral closure $C(D, L)$ of $D$ in $L$ is an intersection of Archimedean valuation domains on $L$.

PROOF. Let $\bar{E}$ be the integral closure of $E$ in $L$. Since $E$ is completely integrally closed, $\bar{E}$ is the complete integral closure of $E$ in $L$ and is a completely integrally closed domain [2, Theorem 13.8]. Clearly $C(D, L) \subseteq \bar{E}$, but since $\bar{E}$ is integral over $E$ it follows that $\bar{E}$ is almost integral over $D$ [2, Corollary 13.2], and hence $\bar{E} \subseteq C(D, L)$. Therefore $C(D, L)=\bar{E}$. By hypothesis $E=\bigcap_{a \in A} V_{a}$, where each $V_{a}$ is an Archimedean valuation domain on $K$, and Lemma 1 shows that $\bar{E}=\bigcap_{a} \overline{V_{a}}$, where bars indicate integral closure in $L$. Now $\overline{V_{a}}$ is a Prüfer domain [2, Theorem 20.1] and $\operatorname{dim} \overline{V_{a}}=\operatorname{dim} V_{a} \leq 1$. Consequently, $\overline{V_{a}}$ is an intersection of Archimedean valuation domains on $L$, so $\bar{E}=\bigcap_{a} \overline{V_{a}}$ is also an intersection of Archimedean valuation domains on $L$. This completes the proof of Theorem 2 .

Suppose $R$ is a subring of $S$ and $S$ is a subring of $T$. In contrast with the situation for integrality, almost integrality is not transitive - that is, $T$ need not be almost integral over $R$ even if $T / S$ and $S / R$ are almost integral. For example, if $D$ is an integral domain with quotient field $K$, the complete integral closure $D^{(1)}$ of $D$ need not be completely integrally closed [3, Example 1]; in fact, Hill [4] has given an example of a Bezout domain $D$ such that $D^{(n)}<D^{(n+1)}$ for each positive integer $n$, where $D^{(n+1)}$ is defined inductively as the complete integral closure of $D^{(n)}$. Another contrast between the properties of integrality and almost integrality is that the inclusion $C(R, S) \subseteq C(R, T) \bigcap S$ may be proper [3, Example 2]. However, it follows from part (b) of [3, Proposition 2] that $C(R, S)=C(R, T) \cap S$ if $S$ is a field. We use this equality in the proof of Proposition 3.

PROPOSITION 3. Suppose $D$ is an integral domain with quotient field $K$ and $T$ is an extension ring of $K$. If $\Delta$ is the integral closure of $K$ in $T$, then $C(D, T)=C(D, \Delta)$.

Proof. By the result from [3] just cited, $C(D, \Delta)=C(D, T) \bigcap \Delta$. Hence, to prove Proposition 3, it suffices to show that $C(D, T) \subseteq \Delta$. This is straightforward: if $t \in C(D, T)$ then clearly $t \in C(K, T)$, and since $K$ is Noetherian, $t$ is integral over $K$ - that is, $t \in \Delta$.

THEOREM 4. Let $D$ be an integral domain with quotient field $K$ and let $L$ be an extension field of $K$. If the complete integral closure of $D$ is an intersection of Archimedean valuation domains on $K$, then the complete integral closure of $D$ in $L$ is an intersection of Archimedean valuation domains on $L$.

Proof. Let $\Delta$ be the algebraic closure of $K$ in $L$. Proposition 3 shows that $C(D, L)=C(D, \Delta)$, and Theorem 2 shows that $C(D, \Delta)$ is an intersection of 
Archimedean valuation domains on $\Delta$. Hence the proof is complete if $\Delta=L$. If $\Delta<L$, we consider two cases.

CASE 1. $C(D, L)=\Delta$. In this case we show that if $t \in L-\Delta$, then there exists a rank-one valuation domain on $L$ that contains $\Delta$, but not $t$. Thus, since $t^{-1}$ is transcendental over $\Delta$, then $V=\Delta\left[t^{-1}\right]_{t^{-1} V\left[t^{-1}\right]}$ is a rank-one valuation domain on $\Delta(t)$ that does not contain $t$. The valuation domain $V$ admits an extension to a rankone valuation domain $W$ on $L$ [2, Proposition 20.5], and $t \notin W$ since $t \notin W \cap \Delta(t)$. Therefore $\Delta$ is the intersection of a family of rank-one valuation domains on $L$. This resolves Case 1.

CASE 2. $C(D, L)<\Delta$. In this case there exists a family $\left\{V_{a}\right\}_{a \in A}$ of rank-one valuation domains on $\Delta$ such that $C(D, L)=\bigcap_{a \in A} V_{a}$. For each $a \in A$, let $W_{a}$ be a rank-one extension of $V_{a}$ to $L$ and, by Case 1 , let $\left\{U_{b}\right\}_{b \in B}$ be a family of rankone valuation domains on $L$ such that $\Delta=\bigcap_{b \in B} U_{b}$. If $\mathscr{F}=\left\{W_{a}\right\}_{a} \bigcup\left\{U_{b}\right\}_{b}$, then $\mathscr{F}$ is a family of rank-one valuation domains on $L$ and $\bigcap \mathscr{F}=\left(\bigcap_{a} W_{a}\right) \cap \Delta=$ $\bigcap_{a}\left(W_{a} \cap \Delta\right)=\bigcap_{a} V_{a}=C(D, L)$.

We remark that the converse of Theorem 4 is also valid: If $C(D, L)=\bigcap_{b \in B} W_{b}$, where each $W_{b}$ is an Archimedean valuation domain on $L$, then $C(D, K)=$ $C(D, L) \bigcap K=\bigcap_{b \in B}\left(W_{b} \bigcap K\right)$, where each $W_{b} \bigcap K$ is an Archimedean valuation domain on $K$ [2, Theorem 19.16]. Thus, for any extension field $L$ of $K$, the condition that $C(D, L)$ is an intersection of Archimedean valuation domains on $L$ depends only upon $D$, not on $L$.

\section{References}

[1] H. S. Butts and W. W. Smith, 'On the integral closure of a domain', J. Sci. Hiroshima Univ. Ser. A-I 30 (1966), 117-122.

[2] R. Gilmer, Multiplicative ideal theory (Queen's Univ., Kingston, 1992).

[3] R. Gilmer and W. Heinzer, 'On the complete integral closure of an integral domain', J. Austral. Math. Soc. 6 (1966), 351-361.

[4] P. Hill, 'On the complete integral closure of a domain', Proc. Amer. Math. Soc. 36 (1972), 26-30.

[5] W. Krull, 'Allgemeine Bewertungstheorie', J. Reine Angew. Math. 167 (1932), 160-196.

[6] M. Nagata, Local rings (Wiley, New York, 1962).

[7] T. Nakayama, 'On Krull's conjecture concerning completely integrally closed integrity domains I, II', Proc. Imperial Acad. Tokyo 18 (1942), 185-187, 233-236.

[8] —_, 'On Krull's conjecture concerning completely integrally closed integrity domains III', Proc. Japan Acad. 22 (1946), 249-250.

Florida State University

Tallahassee, FL 32306-3027

USA

e-mail: gilmer@math.fsu.edu 